\title{
Cryptanalysis of a novel cryptosystem based on chaotic oscillators and feedback inversion
}

\author{
G. Álvarez Marañón, L. Hernández Encinas*, \\ F. Montoya Vitini and J. Muñoz Masqué \\ Instituto Física Aplicada \\ Consejo Superior de Investigaciones Científicas \\ C/ Serrano 144, 28006-Madrid, Spain \\ Emails: \{gonzalo, luis, fausto, jaime\}@iec.csic.es
}

\begin{abstract}
An analysis of a recently proposed cryptosystem based on chaotic oscillators and feedback inversion is presented. It is shown how the cryptosystem can be broken when Duffing's oscillator is considered. Some implementation problems of the system are also discussed.
\end{abstract}

\section{Introduction}

In recent years, a growing number of cryptosystems based on chaos have been proposed 1, many of them fundamentally flawed by a lack of robustness and security. In the Letter [2], the authors have proposed a symmetric cryptosystem based on chaotic oscillators. More precisely, let $N: L_{\infty e}\left(\mathbb{R}_{+}\right) \rightarrow L_{\infty e}\left(\mathbb{R}_{+}\right)$be a non-linear time-varying system, where $L_{\infty e}\left(\mathbb{R}_{+}\right)$stands for the extended $L_{\infty^{-}}$ space on $\mathbb{R}_{+}$, and let $S_{1}$ and $S_{2}$ be two signal generators which generate the time functions $t \mapsto w_{1}(t)$ and $t \mapsto w_{2}(t)$, respectively. The encryption process is defined by adding the signal generator $S_{2}$ to the output of the dynamic evolution of $N$. Explicitly, to encrypt a message defined by a train of pulses $t \mapsto p(t)$, of suitable width and of amplitude zero or one, is necessary to compute the function $u(t)=p(t)+w_{1}(t), \forall t \geq 0$. Then, $u(t)$ is applied to the system $N$ and its output is added to $w_{2}(t)$. The ciphertext is then defined to be $c(t)=(N u)(t)+w_{2}(t)$, $\forall t \geq 0$.

The decryption process consists of the two signal generators, $S_{1}$ and $S_{2}$, and a feedback system $S(g, N)$, where $N$ is the non-linear system used in the encryption protocol and $g$ is the gain of the system. To decrypt a message $c(t)$, one subtracts $w_{2}(t)$ from $c(t)$ and the result is the input to the system $S(g, N)$. Its output, $\widetilde{u}(t)$, is a good, but noisy, approximation of $u(t)$; also, the difference between $w_{1}(t)$ and $\widetilde{u}(t)$ gives $\widetilde{p}(t)$, which is a good, but noisy, approximation of $p(t)$. Using a low-pass filter $L(s)$ and a quantizer $Q$, the original message is obtained. In order to recover the original message exactly, $L(s)$ should be designed carefully. Although the authors seem to base the security of their

\footnotetext{
${ }^{*}$ Corresponding author. Tel. (+34) 915618806 (Ext. 458), Fax: (+34) 914117651
} 
cryptosystem on the chaotic behavior of the output of the non-linear system $N$, no analysis of security is included.

In the present Letter we discuss the weaknesses of this system in Section 2 and we analyze its practical implementation in Section 3

\section{Attacks to the cryptosystem}

In this section we show how to break the cryptosystem proposed in [2] when Duffing's oscillator is used as the non-linear time-varying system [2, §3.1], which, in fact, is the first example explained in detail. The main problem with this cryptosystem lies on the fact that the ciphertext is an analog signal, whose waveform depends on the system parameter values and the plaintext signal. Likewise, the detected signal before the quantizer depends on these same parameters. The study of these signals provides the necessary information to recover a good estimation of the system parameter values and the correct plaintext.

We consider the first example in [2] §4.1], for Duffing's oscillator, represented by:

$$
N: \ddot{x}(t)+\delta \dot{x}(t)-\alpha x(t)+\beta x(t)^{3}=u(t), x(0)=0, \dot{x}(0)=0 .
$$

In their example $w_{1}(t)=A \cos \omega t$ and $w_{2}(t) \equiv 0$. The key of the system is made by the oscillator's parameters $(\delta, \alpha, \beta)$, and the signal generator's parameters $(A, \omega)$. Following the example given, we use a key formed by the following set of parameters:

$$
\alpha=10, \beta=100, \delta=1, A=1.5, \omega=3.76 .
$$

Duffing's oscillator is used operating in the chaotic region. This region is roughly characterized by the following values of the parameters:

$$
3 \leq \alpha \leq 15,40 \leq \beta \leq 250,0.5 \leq \delta \leq 1.7,1 \leq A \leq 2,0.5 \leq \omega \leq 7 .
$$

The sensitivity to the parameter values is so low that the original plaintext can be recovered from the ciphertext using a receiver system with parameter values considerably different from the ones used by the sender. As a consequence, it is very economic to try different combinations of the parameters until a reasonable approximation is reached. Although the parameter values can be obtained with a very accurate precision, their knowledge is not necessary to recover the plaintext.

We have found that the message can be decrypted even when $\beta$ has an error of $\pm 5 \% ; \delta$ has an error from $-30 \%$ to $+60 \%$ and $\alpha$ has an absolute error of \pm 2 integers; with respect to the original set of parameters (2).

In Fig. 1 we show the power spectral analysis of the example ciphertext signal. As is observed, the frequency of the forced oscillator is totally evident. The spectrum highest peak appears at the $S_{1}$ signal generator frequency of $\omega=3.76$. Thus, by simply examining the ciphertext, one of the elements of the key $(\omega)$ is obtained.

Next, the attacker uses a receiver for which $A=0$, and the rest of the 
parameters takes values from the following sets:

$$
\begin{aligned}
\alpha= & \{5,9,13\}, \\
\beta= & \{43,47,51,56,62,68,75,82,91,100,110, \\
& 120,130,145,160,180,200,220,240\}, \\
\delta= & \{0.7,1.3\} .
\end{aligned}
$$

This makes a total of 114 possible combinations, which should be tried one by one. To check whether the choice of the parameters is good, we look at the output of the low-pass filter $L(s)$, which we call $\hat{p}(t)$. When the parameter values are slightly different from the right ones, then $\hat{p}(t)$ will look like a square signal summed with a pure sine. The frequency of this sine corresponds to the value of $\omega$ previously calculated from the spectrum of the ciphertext. The amplitude of this sine corresponds exactly to the value of $A$ used by the sender.

Next, the value of $A$ just computed is used to regenerate the plaintext. Due to the low sensitivity to the parameter values, although the exact values are unknown, the deciphered plaintext signal will be equal (or very close) to the original one. In Fig. 2 the recovered plaintext is depicted for the following parameter values:

$$
\alpha=9, \beta=100, \delta=0.7, A=1.4, \omega=3.76 .
$$

The first three values are taken from the equations (4)-(6). Although the parameter errors are $10 \%, 0 \%, 30 \%, 6.66 \%$, and $0 \%$, respectively, the plaintext is correctly recovered. These values could be further refined by varying them in an effort to approximate $\hat{p}(t)$ to a square wave.

\section{Difficulties of practical implementation}

In this section we discuss the difficulties that this cryptosystem will face if it is practically implemented.

\subsection{Analog transmission}

The proposed cryptosystem seems to present serious problems in a real transmission, because the recovered signal at the receiving end of the transmission path will be very difficult to decrypt.

Apparently, the authors have only implemented a software simulation of the complete encryption/decryption system, feeding the ciphertext (the output generated by the encryption system) directly as the input to the decryption system. The generators $S_{1}$ and $S_{2}$, part of the system key, look to be connected simultaneously and locally to both the encryption and decryption systems.

In real world applications, however, things happen in a very different way. Ideal transmission lines introduce an unknown amount of attenuation and delay in the transmitted signal. Furthermore, real transmission lines introduce distortion and noise too. Moreover, wireless communication systems exhibit time-variable attenuation and delay.

Thus, the input signal at the receiver end $c^{\prime}(t)$ and the transmitted signal $c(t)$ will differ. In the most favorable case, if we assume that we are using an ideal line, the received signal will be $c^{\prime}(t)=k c(t+\tau)$, were $k$ and $\tau$ are the attenuation and delay of the line. 


\subsubsection{Synchronization}

As the authors point out, most continuous chaotic cryptosystems described until now are based on the synchronization of two chaotic systems. The claimed novelty of the present cryptosystem relies on the lack of synchronization between encryption and decryption; but this is an erroneous claim, because in the software simulations the authors have used a hidden synchronization mechanism consisting of the local and simultaneous connection of generators $S_{1}$ and $S_{2}$ to both the encryption and decryption systems.

In real world applications, given that transmission lines have limited bandwidth, when transmitting to a remote system, signal delay will take place. The delay amount may vary for different frequency components of the signal, depending on the line impulsive response. Thus, the observed waveforms at sender and receiver ends may differ and it will be very difficult to estimate the right moment to start the receiving generators.

Some measures should be taken to assure that both ends are using signal generators $S_{1}$ and $S_{2}$ with exactly the same phase in respect to the ciphertext. However, no mesure is considered by the authors. Hence the receiver end's generators will never generate the adequate signal.

\subsubsection{Attenuation}

Another factor to be considered is the line attenuation. No continuous transmission or storage system (cable, optical, magnetic or wireless) grants that the received or reproduced signal amplitude preserves the original amplitude. If the signal is transmitted over a switched network, the attenuation will change each time that a new connection is made. If the signal is transmitted over a wireless channel, the attenuation will vary depending on changing atmospheric conditions, changing reflections, and changing multipath.

When transmitting a signal of known constant amplitude (e.g. square pulses or frequency modulated sinusoids) it is possible to equalize the received signal, restoring the correct amplitude level. But in the present case, as the signal is chaotic, its amplitude is varying in an unpredictable fashion, so it is impossible any level restoring.

Therefore, it will be impossible to subtract exactly $w_{2}$ at the receiver end. Hence, the signal $r$ at the input of the decryption system feedback loop will be $r(t)=c^{\prime}(t)-w_{2}(t)$, i.e.:

$$
r(t)=k(N u)(t+\epsilon)+k w_{2}(t+\epsilon)-w_{2}(t),
$$

where $\epsilon$ is the time inaccuracy in the determination of the right moment to start the receiving generators. Hence, the decrypted signal will be:

$$
y(t) \approx\left(N^{-1} r\right)(t)=\left(N^{-1}\left(k(N u)(t+\epsilon)+k w_{2}(t+\epsilon)-w_{2}(t)\right)\right)(t) .
$$

As $N$ is a nonlinear chaotic map and due to the sensitive dependence on the initial conditions that characterize chaos [3, p. 119], the decrypted signal $y(t)$ will never match the plaintext $p(t)$.

The recovered plaintext errors induced by the use of a real communication channel with restricted bandwidth, attenuation and/or noise are illustrated in Fig. 3 . 
Moreover, the authors seem to base the security of their cryptosystem on the chaotic behavior of $N$, although no evidence of that is shown. In any case, the chaotic profile of the output $x(t)$ in Duffing's oscillator (11) is not always guaranteed for every input $u(t)=p(t)+w_{1}(t)$, even in the chaotic range (3), and the sensitive dependence on the initial conditions is diminished as they are kept to be fixed, $x(0)=0, \dot{x}(o)=0$, in Duffing's equation (11).

\subsection{Digital transmission}

If a discretization of the ciphertext is sent instead of the dynamic evolution of the system $N$, then there are two options.

In the first one, the ciphertext is discretized only at the nodes $i=0, \ldots, n$, where $n$ is the number of pulses of $p(t)$. Then, the ciphertext sent by the sender must be the 3 -uples $\left(x\left(t_{i}\right), \dot{x}\left(t_{i}\right), \ddot{x}\left(t_{i}\right)\right), i=0, \ldots, n$, since the receiver needs to know these values - and not only the $x\left(t_{i}\right)$-in order to be able to decrypt the message, as the usual methods of discretization do not allow to obtain the values of the derivative at the nodes $t_{i}$ in terms of the values of the function at such nodes. For example, if one uses the Runge-Kutta method (see [4, §163]) to solve $\ddot{x}=f(t, x, \dot{x})$, then the values of the first derivative are given by

$$
\begin{aligned}
\dot{x}\left(t_{0}+h\right) & =\dot{x}\left(t_{0}\right)+\frac{1}{6}\left(k_{1}+2 k_{2}+2 k_{3}+k_{4}\right), \\
k_{1} & =h f\left(t_{0}, x\left(t_{0}\right), \dot{x}\left(t_{0}\right)\right), \\
k_{2} & =h f\left(t_{0}+\frac{1}{2} h, x\left(t_{0}\right)+\frac{1}{2} h \dot{x}\left(t_{0}\right)+\frac{1}{8} h k_{1}, \dot{x}\left(t_{0}\right)+\frac{1}{2} k_{1}\right), \\
k_{3} & =h f\left(t_{0}+\frac{1}{2} h, x\left(t_{0}\right)+\frac{1}{2} h \dot{x}\left(t_{0}\right)+\frac{1}{8} h k_{2}, \dot{x}\left(t_{0}\right)+\frac{1}{2} k_{2}\right), \\
k_{4} & =h f\left(t_{0}+h, x\left(t_{0}\right)+h \dot{x}\left(t_{0}\right)+\frac{1}{2} h k_{3}, \dot{x}\left(t_{0}\right)+k_{3}\right) .
\end{aligned}
$$

We remark on the fact that the values for the second derivative should be computed from the formula

$$
\ddot{x}\left(t_{i}\right)=f\left(t_{i}, x\left(t_{i}\right), \dot{x}\left(t_{i}\right)\right),
$$

where $f$ is the function defining the dynamic system $N$. This fact implies that the transmission has a high factor expansion as every pulse of the original message is transmitted by means of a 3 -uple of real numbers with a consistent number of decimals.

The second option consists in computing a much more long list of values $x\left(s_{i}\right), i=0, \ldots, m$, with $m \gg n$. In this case, the values for the first derivative can be obtained from the formulas above; but the second derivative should also be included in the transmission. Hence, in this case the ciphertext is $\left(x\left(s_{i}\right), \ddot{x}\left(s_{i}\right)\right), i=0, \ldots, m$. What is gained in not sending the first derivative is lost by the greater number of entries of the list.

In any case, the values of the first and second derivatives cannot be computed by the usual approximate formulas

$$
\begin{aligned}
& \dot{x}\left(t_{i}\right) \approx \frac{x\left(t_{i+1}\right)-x\left(t_{i}\right)}{h}, \\
& \ddot{x}\left(t_{i}\right) \approx \frac{x\left(t_{i+2}\right)-2 x\left(t_{i+1}\right)+x\left(t_{i}\right)}{h^{2}},
\end{aligned}
$$


as they produce considerable errors in the decryption process due to the nonlinear terms in $N$.

\section{Conclusion}

As a consequence of the previous analysis, the cryptosystem studied cannot work in practice because it is not using any synchronization mechanism and because it is not robust to real channel conditions. On the other hand, the cryptosystem is rather weak, since it can be broken by using a set of 114 parameter values only. The total lack of security, along with the lack of robustness, discourages the use of this algorithm for secure applications.

Acknowledgements This work is supported by Ministerio de Ciencia y Tecnología of Spain, research grant TIC2001-0586.

\section{References}

[1] T. Yang, A Survey of Chaotic Secure Communication Systems. International Journal of Computational Cognition 2 (2003), 81-130.

[2] S. M. Shahruz, A. K. Pradeep, R. Gurumoorthy, Design of a novel cryptosystem based on chaotic oscillators and feedback inversion. Journal of Sound and Vibration 250 (2002), 762-771.

[3] R. L. Devaney, A first course in chaotic dynamical systems, AddisonWesley, Reading, MA, 1992.

[4] D. Zwillinger Handbook of differential equations, Academic Press, Inc., Boston, 1989. 


\section{Figure captions}

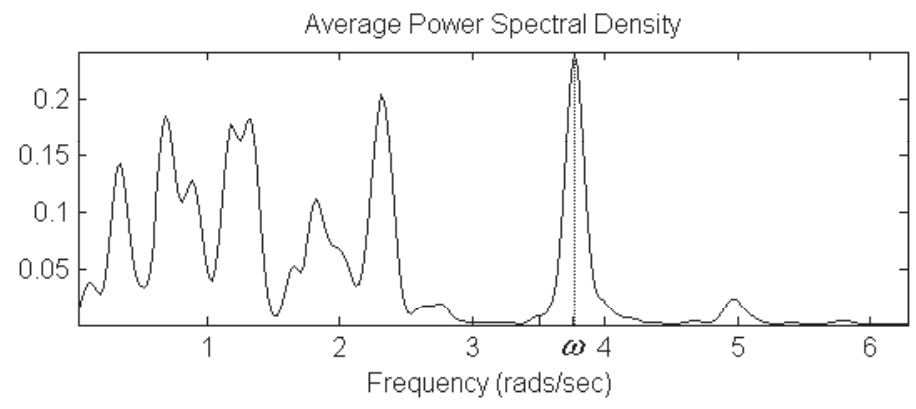

Figure 1: Power spectral analysis of the ciphertext signal. The highest peak corresponds to the frequency of $S_{1}$ and lies at $\omega \approx 3.76$. 

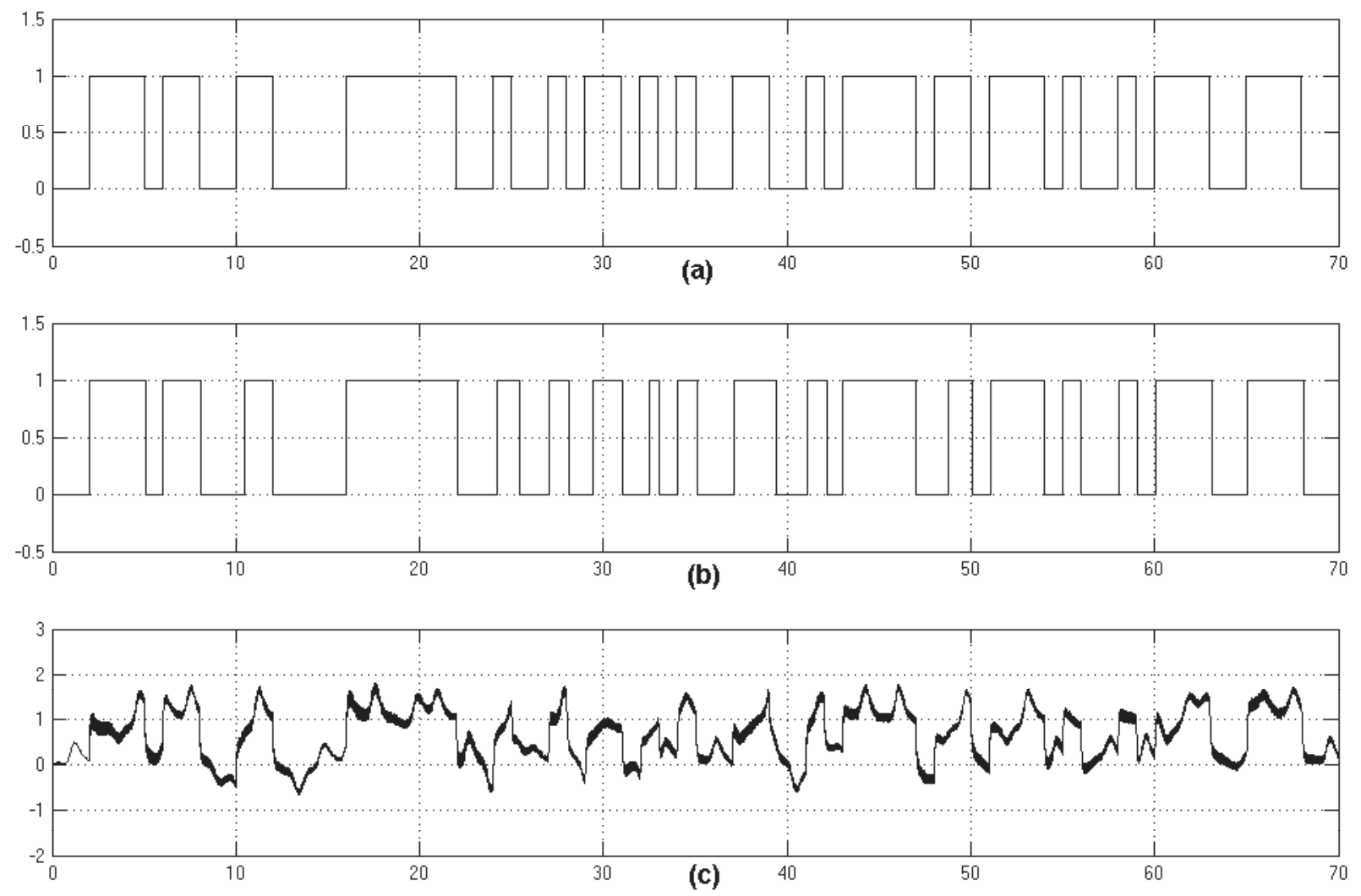

Figure 2: Plaintext recovery with inexact receiver parameter values. Time histories of: (a) plaintext; (b) recovered plaintext; (c) $\hat{p}(t)$. 

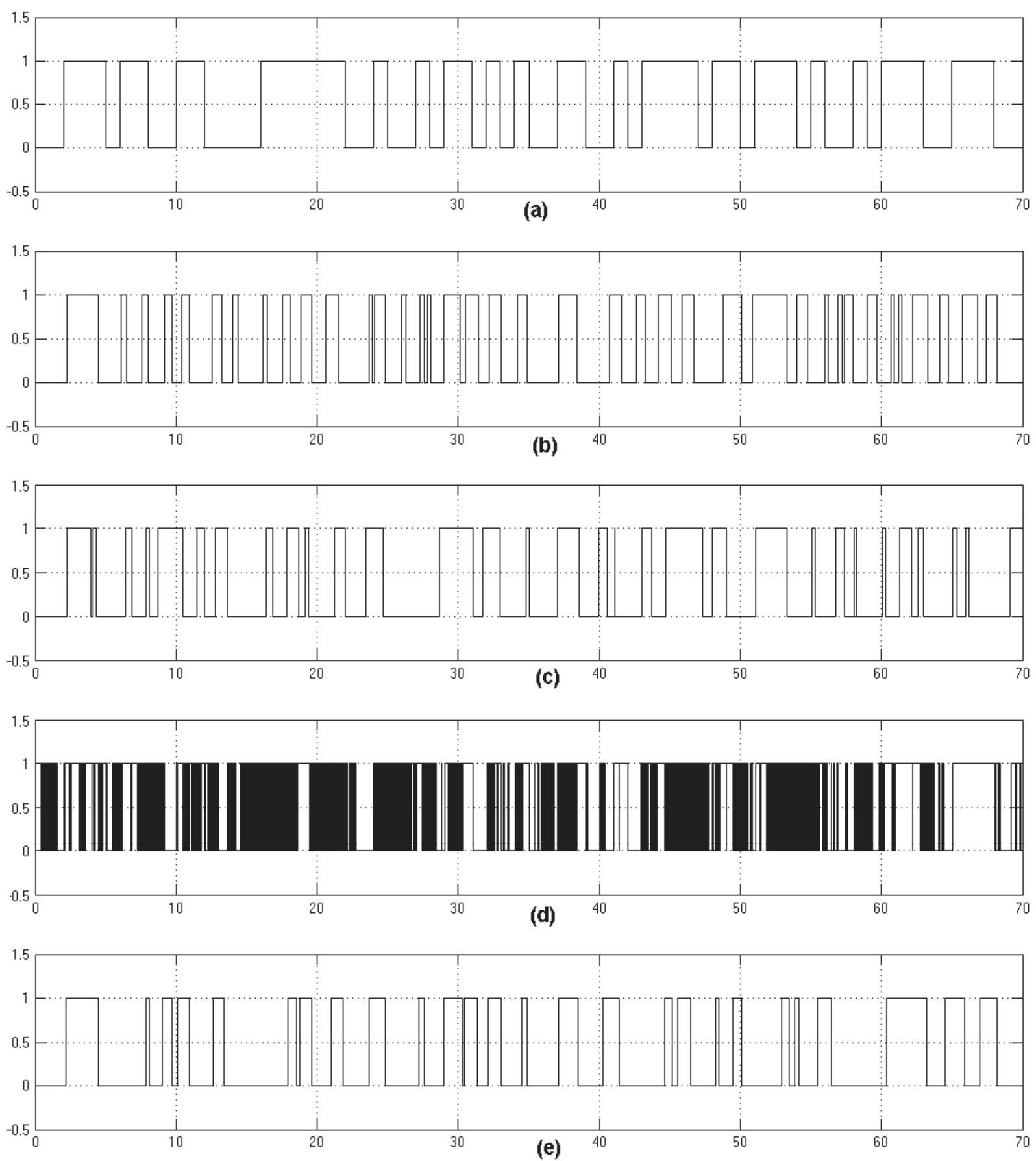

Figure 3: Effects of a real communication channel: (a) plaintext; (b) recovered plaintext with channel bandwidth restricted to $\omega=6.28 \mathrm{rad} / \mathrm{seg}$; (c) recovered plaintext with channel attenuation of $3 \mathrm{~dB}$; (d) recovered plaintext with channel noise of $-40 \mathrm{~dB}$; (e) recovered plaintext with channel bandwidth restricted to $\omega=9.42 \mathrm{rad} / \mathrm{seg}$, attenuation of $0.5 \mathrm{~dB}$ and noise of $-50 \mathrm{~dB}$. The parameter values at the sender and receiver ends match exactly. 